\title{
Germanica
}

unifiée

\section{Fonction identitaire de l'écriture chez Jakob Hein}

Identitätsstiftende Literatur bei Jakob Hein

\section{Hélène Yèche}

\section{OpenEdition}

\section{Journals}

Édition électronique

URL : http://journals.openedition.org/germanica/609

DOI : 10.4000/germanica.609

ISSN : 2107-0784

Éditeur

Université de Lille

\section{Édition imprimée}

Date de publication : 1 juin 2009

Pagination : $51-63$

ISBN : 978-2-913857-23-0

ISSN : 0984-2632

\section{Référence électronique}

Hélène Yèche, «Fonction identitaire de l'écriture chez Jakob Hein », Germanica [En ligne], 44 | 2009, mis en ligne le 01 juin 2011, consulté le 10 décembre 2020. URL : http://journals.openedition.org/ germanica/609; DOI : https://doi.org/10.4000/germanica.609

Ce document a été généré automatiquement le 10 décembre 2020.

(C) Tous droits réservés 


\title{
Fonction identitaire de l'écriture chez Jakob Hein
}

\author{
Identitätsstiftende Literatur bei Jakob Hein
}

Hélène Yèche

1 Depuis ses débuts littéraires sur le ton léger de l'autodérision avec Mein erstes T-Shirt (Mon premier T-shirt) (Piper, 2001) et Formen menschlichen Zusammenlebens (Formes de cohabitation humaine) (Piper, 2003) jusqu'à un premier roman sobre et émouvant consacré à la mort de sa mère Vielleicht ist es sogar schön (Peut-être même que c'est beau) (Piper, 2004), Jakob Hein ne cesse de ressasser, commenter et interroger l'histoire familiale à travers sa fiction. Né en 1971 à Leipzig et fils d'un écrivain renommé ${ }^{1}$, il fait partie des jeunes auteurs de l'Est dont l'écriture, vingt ans après la Chute du Mur et la réunification allemande, se nourrit pour une part essentielle du passé récent. Mais le lecteur qui découvre l'œuvre de Jakob Hein est surtout frappé par l'enracinement de la pratique de l'écriture au sein de la cellule familiale, qui revient comme un leitmotiv dans la narration. Loin de refouler un passé jugé plus amusant qu'encombrant, Jakob Hein fait de l'histoire familiale le terreau naturel de son inspiration. Le ton est toujours vif et lucide, plein de tendresse et d'humour. Le dialogue entre les générations, chez ce jeune écrivain de l'Allemagne réunifiée, est immanent et constitutif d'une écriture autobiographique centrée sur le noyau familial dont il convient d'explorer l'impérieuse nécessité identitaire.

\section{Écriture autobiographique}

2 Les œuvres d'auteurs d'origine est-allemande nés dans les années 1970 qui reviennent sur leur passé en RDA sont loin de constituer une exception, comme l'a bien analysé Carola Hähnel-Mesnard ${ }^{2}$. La littérature de la "dernière " génération de la RDA, des Zonenkinder (enfants de la zone) comme on les appelle depuis la parution du livre éponyme de Jana Hensel en 2002, oscille entre récit d'enfance, souvenir, autobiographie et témoignage, sans qu'une catégorisation exacte soit toujours possible. 
3 Les premiers ouvrages de Jakob Hein ne dérogent pas à cette règle: même s'ils représentent un corpus à part, clairement focalisé sur les dernières années d'existence de la $\mathrm{RDA}$ et les premières expériences post-réunification dont le caractère autobiographique semble ne faire aucun doute, leur nature mérite examen. Car pour ces trois premiers ouvrages, aucun terme n'indique une quelconque appartenance à un genre littéraire établi ${ }^{3}$. Il faut se plonger dans la lecture du livre pour savoir de quelle catégorie l'écrit relève. Aucune indication ne figure sur la couverture ou dans les premières pages: tout au plus l'éditeur se permet-il d'avancer quelques remarques formelles, afin probablement de guider le lecteur dans l'aventure. Ainsi pour le premier ouvrage de Jakob Hein, une note éditoriale insiste sur le terme Geschichten (histoires) : l'auteur depuis l'enfance aime raconter des histoires et c'est manifestement ce qu'il fait dans les pages qui suivent. Il est intéressant de noter d'emblée que ces histoires ne sont pas présentées comme un récit autobiographique : au contraire, l'éditeur souligne indirectement leur nature fictionnelle dans la mesure où il termine sa brève introduction en précisant que, "dans la vraie vie ${ }^{4}$ ", Jakob Hein est médecin et vit à Berlin avec sa famille. Il s'instaure donc une distance naturelle entre ces histoires et l'histoire de la vie de l'auteur. D'ailleurs, comme le fait remarquer un critique, le livre de Jakob Hein raconte " les épisodes joyeux d'une sombre jeunesse en RDA ", insistant sur «ce qui s'est réellement passé » ou sur «ce qui aurait pu se passer $»^{5}$ comme l'indiquent les titres complémentaires des deux derniers récits du volume. Pour Elke Brüns, le texte de Jakob Hein ne relève en rien de la « littérature autobiographique du souvenir", il s'agit plutôt d'une littérature ironique qui met un point final autoréférentiel à la mémoire collective et littéraire de la RDA'

4 Toutefois les choses ne sont pas aussi simples, car Jakob Hein est en même temps présenté d'emblée au lecteur par l'éditeur comme «le jeune héros ${ }^{7}$ » du texte dans le cas de Mein erstes T-Shirt. De plus, la préface allographe ${ }^{8}$ de Wladimir Kaminer à ce même ouvrage - intitulée de façon parodique Mein erster Jakob Hein (Mon premier Jakob Hein) - revient avec insistance sur le portrait du jeune écrivain dans l'intimité, soulignant à l'intention des lecteurs le caractère insaisissable et polymorphe du personnage tel qu'il se dégage des différents épisodes de son livre ${ }^{9}$. Ce faisant, W. Kaminer croise en permanence la perspective autobiographique et la transposition fictionnelle, tout en infléchissant nettement la lecture dans un sens autoréférentiel. Il ressort également du discours éditorial que l'ouvrage suivant repose sur les expériences de voyage de l'auteur parti à la découverte du Nouveau Monde ${ }^{10}$. Quelques années plus tard, le même éditeur n'hésitera pas à ranger les débuts littéraires de Jakob Hein dans la catégorie des écrits autobiographiques ${ }^{11}$. Force est de constater ici le rôle déterminant de l'éditeur et du péritexte allographe en général dont les remarques introductives au fil des éditions tendent à influencer la lecture référentielle de l'œuvre. En se focalisant sur la présentation de la personne de l'écrivain, le discours éditorial a tendance à tirer le texte dans le registre référentiel. Si l'on se réfère aux travaux de Philippe Lejeune sur l'autobiographie ${ }^{12}$, il y a ici identité avérée entre l'auteur, le narrateur et le personnage, condition impérative de l'écriture autobiographique. Ce " pacte autobiographique » a pour corollaires un mode d'énonciation, le récit à la première personne, et une structure narrative, le récit rétrospectif, qui caractérisent le genre et que l'on retrouve effectivement présents dans les trois ouvrages. Dans l'une des anecdotes sur sa jeunesse en RDA comme dans l'un des récits de son voyage aux USA, le narrateur-auteur utilise même son prénom et son nom comme signe 
d'identification irréfutable ${ }^{13}$. L'identité onomastique qui fonde le protocole propre à l'autobiographie est donc soulignée par l'auteur désireux de se faire reconnaître.

5 Les premiers textes de Jakob Hein affichent à l'évidence de nombreux indices d'autobiographie. Pourtant, en dépit de ces prises de position référentialistes, jamais l'auteur ne semble vouloir s'attacher à définir plus avant la perspective adoptée. Oscillant entre souvenirs d'enfance, impressions de voyage et chronique familiale, sous le signe d'une littérature pop caractéristique des jeunes écrivains de l'Allemagne unifiée $^{14}$, les livres de Jakob Hein s'imposent comme des évidences, sans que l'auteur ressente le besoin de justifier ou d'expliquer sa démarche. Des réflexions sur le statut et la véracité du souvenir, ou sur la fiabilité de la mémoire, fréquentes dans le genre autobiographique, sont inexistantes dans la littérature qui nous intéresse sans qu'il faille pour autant en conclure au souvenir brut, sans aucune mise à distance. Car c'est au contraire sur une démarche ironique confinant à l'autodérision que repose la poétique de Jakob Hein.

6 Dix ans après la chute du Mur de Berlin, Jakob Hein parvient à s'imposer sur la scène littéraire avec un recueil d'anecdotes tirées du quotidien de son enfance et adolescence est-allemandes, salué par la critique comme une série de miniatures qui révèlent autant de visions pathologiques de l'ancienne $\mathrm{RDA}^{15}$. Psychiatre de formation et écrivain à ses heures, l'auteur déclare qu'il n'aurait jamais pu écrire un livre sur la RDA en général : il ne peut écrire qu'à partir de sa propre expérience, de son vécu personnel ${ }^{16}$. De la même façon, son deuxième ouvrage résulte d'une aventure individuelle : de New York à San Francisco, l'auteur a étudié ce qu'il qualifie de "formes de cohabitation humaine ». Mais derrière le récit d'expériences individuelles, et malgré la présence insistante de «trente photographies de l'auteur » illustrant l'ouvrage, se dessine pour le lecteur une technique d'écriture fondée sur l'observation minutieuse et ironique du monde, dans laquelle chacun peut se retrouver. Le troisième livre enfin est consacré à la mort de sa mère et constitue " un portrait de famille autobiographique ${ }^{17}$ ", mais parvient - au-delà du traumatisme individuel - à dégager les lignes de force d'une écriture universelle. Comme le résume fort justement Jörg Magenau : « Avec Vielleicht ist es sogar schön, Jakob Hein a une fois de plus écrit une non-fiction très littéraire le concernant. Tout y est «vrai», rien n'est inventé. ${ }^{18}$ "

7 La littérature de Jakob Hein se situe ainsi d'emblée dans la veine de l'écriture autobiographique au sens noble du terme que l'histoire littéraire lui a longtemps dénié ${ }^{19}$ : mettant en avant l'exemplarité du cas individuel et particulier, Hein se positionne dès les premiers mots de son tout premier ouvrage comme un cas exemplaire, selon le principe d'universalité de l'art dans la définition d'Aristote ${ }^{20}:$ " Es begann bei mir wie bei den meisten, es begann mit einer Gitarre " (Ça commença pour moi comme pour les autres, ça commença par une guitare) ${ }^{21}$. Le "ça " dévoile immédiatement au lecteur le projet autobiographique rétrospectif : ce dont il va être question, c'est de l'histoire d'une vie, d'une existence qui se veut ordinaire ainsi que l'indique le parallèle entre le « je narratif » et les autres. Mais en même temps, ce début de roman fait écho à un modèle célèbre, le "Voyage au bout de la nuit » de Louis-Ferdinand Céline (1932) qui s'ouvre à l'identique sur une quête des origines : "Ca a débuté comme ça ». Ici le "ça » célinien constitue la clé qui ouvre résolument l'espace du roman qui va se révéler transposition du vécu, de la vie recomposée par l'œuvre. Il semble qu'il en aille de même chez Jakob Hein. L'écrivain ne pose pas comme nécessaire une identité entre 
auteur, narrateur et personnage de la narration : son projet littéraire croise son projet autobiographique et s'en nourrit.

\section{Écriture familiale}

Médecin à Berlin, Jakob Hein met en scène des figures presque exclusivement autobiographiques qui lui servent, semble-t-il, à mieux cerner sa propre histoire. Souvenirs d'enfance d'assistantes maternelles peu amènes et de professeurs "entêtés jusqu'à la mort ", souvenirs d'adolescent confronté au Mur de Berlin et aux premières interrogations politiques, souvenirs d'adulte frappé par la maladie et la mort d'un proche nourrissent ainsi tour à tour l'écriture de ce jeune auteur qui a grandi dans l'ancienne RDA. «Enfant j'écrivais pour parler » confie Emmanuel Adély (né en 1962), jeune romancier français dont les quatre premiers romans forment une sorte de quadriptyque familial, évoquant la famille entière, la maison, le couple ou encore la recherche du père et de la mère. Écrire pour communiquer, pour instaurer la relation au monde et aux autres semble également la motivation première pour Jakob Hein qui confie lui aussi une même valeur thérapeutique à l'écriture ${ }^{22}$. Mais sa littérature n'en devient pas pour autant une littérature exclusive du "moi », où le «je narratif » s'épuiserait dans la spirale obsessionnelle de l'autobiographie. Avant de se nourrir de l'histoire familiale, l'écriture chez Jakob Hein prend littéralement naissance dès les premières lignes de la biographie de l'auteur.

Ce n'est donc pas un hasard si, parmi les nombreux indices d'autobiographie présents dans les textes de Jakob Hein, la référence au milieu familial joue un rôle particulièrement prégnant. Plusieurs épisodes «fondateurs » de la vocation d'écrivain du jeune Jakob Hein sont ainsi décrits dans ses premiers ouvrages, formant un discours auctorial référentialiste au sein même du texte. Le quatrième chapitre du livre consacré à la mère s'ouvre par exemple sur un épisode de l'enfance de l'auteur qui offre au lecteur l'image presque idyllique d'une famille d'écrivains où chacun, sans exception, se penche sur sa table de travail : «In allen Zimmern bot sich das gleiche Bild: Eines der Familienmitglieder saß am Schreibtisch.» (Dans toutes les chambres c'était la même image : un des membres de la famille était assis à son bureau ${ }^{23}$. La mère, Christiane Hein, travaille comme scénariste pour la DEFA, le père Christoph Hein est écrivain et le grand frère Georg fait ses devoirs. Le jeune Jakob, alors à peine âgé de quatre ans, est dès l'enfance confronté à un modèle qui va s'imposer comme un impératif : de retour dans sa chambre, l'enfant se met à tracer des lignes de toutes les couleurs sur du papier, en prenant bien soin de placer quelques points ça et là, et une fois la page remplie de ces signes magiques, il passe à la suivante tout en se racontant de merveilleuses histoires de dragons, de princesses et d'Indiens. Son travail accompli, il se rend dans le bureau de sa mère pour lui annoncer fièrement qu'il a écrit une histoire et de la lui lire à haute voix, aussi fidèlement qu'il l'a inventée quelques instants auparavant, respectant les points et les virgules et tournant les pages au moment opportun. Les encouragements précoces de la mère et du père ont probablement influé de manière décisive sur la future carrière de leur fils. Jakob Hein semble ne pas avoir eu d'autre choix que de se conformer au modèle familial. Si durant l'adolescence aucun désir d'écriture ne se manifeste ouvertement, le milieu studieux de cette famille d'écrivains semble peser sur le jeune Jakob comme en témoigne une remarque en passant sur la présence continue de ses parents à la maison ${ }^{24}$ ou encore ce jugement 
péremptoire sur l'activité littéraire du père : « Da mein Vater den ganzen Tag nichts als lesen und schreiben zu tun schien... » (Comme mon père semblait ne rien faire d'autre de ses journées que lire et écrire... ${ }^{25}$ et enfin cet irrésistible portrait de ses géniteurs croqués en dissidents de l'intérieur: "Ich wuchs in einem Haushalt notorischer Nestbeschmutzer auf. Meine Eltern hatten beide Philosophie studiert, nur um jetzt alles besser zu wissen als die Partei- und Staatsführung. " (J'ai grandi dans un ménage de détracteurs notoires. Mes parents avaient tous deux étudié la philosophie uniquement dans le but d'en savoir plus que la direction du Parti et le gouvernement) ${ }^{26}$.

Les souvenirs d'enfance relatant l'expérience de l'écriture et de la lecture constituent également un épitexte auctorial récurrent dans les entretiens avec l'auteur. Jakob Hein se souvient volontiers de ses premières tentatives éditoriales : à l'âge de huit ans, il aurait composé l'une des revues underground les plus confidentielles de la RDA : «Jede Woche schrieb ich sie in ein unauffällig grünes, abwaschbares Ringbuch ...» (Chaque semaine j'écrivais dans un classeur lavable d'un vert passe-partout ${ }^{27}$. Il débute d'ailleurs son deuxième livre par une référence autobiographique à son expérience de lecteur assidu qui devait se cacher sous les couvertures, armé d'une lampe de poche achetée pour l'occasion, afin de pouvoir lire en toute tranquillité, échappant ainsi à l'injonction parentale du «Licht aus!» (Éteins la lumière!) ${ }^{28}$. Et même si l'expérience n'est guère concluante puisque les conditions de lecture s'avèrent trop difficiles et incitent plutôt à la rêverie ${ }^{29}$, on note dans cette mise en scène de l'enfant-lecteur un rapport essentiel à l'univers littéraire qui se poursuit plus loin par la découverte d'un livre d'anglais qui sera à l'origine de la passion du jeune garçon pour les États-Unis : "Zuhause fand ich ein echtes englisches Buch, das natürlich mein Lieblingsbuch wurde. » (Je découvris à la maison un authentique livre anglais qui devint mon livre préféré) ${ }^{30}$. Et finalement, le lecteur apprend aussi qu'un réel projet d'écriture s'est fait jour au moment même du départ pour le nouveau monde puisque le désir d'écriture s'est clairement manifesté la veille : « Kurz vor dem Einschlafen fiel mir noch ein, etwas zum Schreiben mitzunehmen. Ich stand auf, fand nach etwas Kramen einen alten Terminplaner, den ich nie benutzt hatte und packte ihn zusammen mit einem Kugelschreiber in die Tasche.» (Juste avant de m'endormir, je réalisai qu'il me fallait emporter de quoi écrire. Je me levai et trouvai en fouillant un peu un vieil agenda non utilisé que je mis dans mon sac avec un stylo bille) ${ }^{31}$. La suite de l'ouvrage ne comporte aucune autre mention concernant le travail d'écriture, de prise de notes, de transposition du vécu: les anecdotes sur l'expérience américaine de l'auteur s'enchaînent et se succèdent avec naturel jusqu'à l'épisode final du retour à Berlin qui se termine par une allusion à peine voilée à la distance pourtant manifeste entre réalité et fiction : «Langsam wurde es Herbst, und die Amerikaner verließen wieder die Stadt. Ich bekam meine Wohnung zurück und endlich etwas zu tun. Aber das ist eine ganz andere Geschichte.» (L'automne finit par s'installer et les Américains quittèrent la ville. Je récupérai mon appartement et de quoi m'occuper. Mais ceci est une autre histoire $)^{32}$. La conclusion de l'ouvrage confirme in fine au lecteur le statut fictionnel du narrateur. Mais la motivation première de l'écriture, l'envie et le plaisir de raconter, n'en demeure pas moins ancrée dans le passé personnel de l'écrivain.

11 Comme l'indique systématiquement le discours éditorial de l'édition de poche Piper, l'écriture de Jakob Hein obéit au désir de l'enfant de raconter des histoires, de se raconter ainsi qu'en témoigne les nombreuses autoréférences dans le titre des anecdotes du premier volume: "Warum ich Antikommunist wurde» (Pourquoi je devins anticommuniste), «Meine private Hölle» (Mon enfer personnel), «Als wir uns 
das erste Mal besaufen wollten " (La première fois qu'on a voulu se saouler), "Sex in meiner Kindheit » (Le sexe dans mon enfance), « Die Mauer in meinem Kopf » (Le Mur dans ma tête ${ }^{33}$... et c'est encore une histoire personnelle, familiale qui est à l'origine du troisième livre de l'écrivain: "Jakob Hein erzählt die Geschichte eines langsamen Abschiedes und verbindet die literarische Erinnerung an seine Mutter mit dem Porträt einer außergewöhnlichen Familie » (Jakob Hein raconte l'histoire d'un long et lent adieu, mêlant au souvenir littéraire de sa mère le portrait d'une famille hors du commun) $)^{34}$.

La famille, autant que l'histoire personnelle de l'écrivain, constitue le matériau initial d'une littérature en quête d'identité. La présence des parents, et de la mère en particulier, est récurrente dans le triptyque autobiographique des débuts de l'écrivain et c'est précisément la convocation systématique de l'entourage familial, des parents au grand frère en passant par les grands-parents et l'oncle de l'ouest, qui confère à la littérature des débuts de Jakob Hein un ancrage autobiographique indéniable. Si le rapport au père demeure plus discret, il intervient cependant au moment crucial où le fils doit assumer sa vocation littéraire et donc le caractère intergénérationnel de son écriture, qui est ici d'autant plus flagrant que l'on se trouve en présence d'une écriture à la fois issue et nourrie de l'héritage familial. Jakob Hein raconte dans un entretien qu'il a envisagé un bref instant de prendre un pseudonyme pour éviter de répondre à l'éternelle question des origines en sa qualité de fils d'un écrivain connu. Mais il s'est ravisé, arguant du fait que «Hein est un nom agréable car court et facile à retenir " 35 . Assumant pleinement ses origines familiales, Jakob Hein tente d'exorciser par l'écriture personnelle un autre passé, celui d'un pays disparu, devenu caduc suite aux bouleversements historiques de l'automne 1989.

\section{Écriture testimoniale}

Tournée vers l'extérieur du moi, vers l'extériorité, l'écriture autobiographique cherche aussi à se réapproprier un monde perdu pour comprendre le monde présent, et c'est probablement ainsi qu'il faut interpréter la littérature autobiographique de Jakob Hein à ses débuts. Après la rupture majeure que représente la chute du régime communiste et l'effondrement de la RDA, le retour sur le passé de l'Allemagne socialiste constitue une étape capitale et quasi obligée pour le jeune écrivain dans le contexte de l'Allemagne unifiée. Comme chez d'autres auteurs de la génération "Mauerfall ${ }^{36}$ ", notamment Thomas Brussig, Jana Simon, Jana Hensel ou encore Claudia Rusch, le quotidien vécu en RDA se trouve au centre des récits de Jakob Hein. Ce sont des souvenirs personnels ordinaires à la maison, à l'école ou avec les amis qui forment une sorte d'album de famille que l'auteur feuillette avec une nostalgie distanciée. L'État socialiste, qui fut le cadre de l'enfance et de la jeunesse de l'auteur, se trouve en quelque sorte réhabilité car transfiguré par l'espace du souvenir. Le fait de raconter la RDA d'un point de vue strictement personnel et particulier ôte tout caractère traumatique à la résurgence du passé est-allemand comme le note Elke Brüns qui parle à ce propos de "nostalgische Verklärung " (transfiguration nostalgique) ${ }^{37}$. L'écriture sur fond autobiographique revêt pour Jakob Hein une fonction thérapeutique et salvatrice, qui s'apparente au travail de deuil : c'est indéniablement le cas du troisième livre de l'auteur écrit immédiatement après le décès de sa mère, mais il en va de même pour les deux premiers ouvrages, Mein erstes T-Shirt étant un adieu à l'enfance socialiste 
tandis que l'expérience américaine relatée dans Formen menschlichen Zusammenlebens relève de la transition d'un système politique à un autre, du passage au Wunderwelt capitaliste comme l'annonçait déjà le narrateur à la fin du premier recueil d'anecdotes : "Ich [...] ging auf die Suche nach neuen Lebenszielen in der Wunderwelt des Kapitalimus" (Je partis à la recherche de nouvelles raisons de vivre au pays merveilleux du capitalisme) $)^{38}$. Les débuts littéraires de Jakob Hein obéissent à une démarche transitionnelle menant du retour sur l'enfance et la perte de la mère à la découverte et à l'exploration de l'univers post-socialiste.

Les souvenirs de Jakob Hein sont amusants et désuets. Essentiellement autoréférentiels, ils ne semblent pas viser un quelconque objectif. Parfois pourtant l'écrivain paraît vouloir conférer une valeur testimoniale à son écriture, en rappelant discrètement au lecteur la dureté ou le caractère absurde du régime totalitaire : «Besonders schwer war es in der DDR " (La vie était particulièrement difficile en RDA), "Es war eine graue Zeit " (C'était une période triste), «Als ich ein Kind war, da hatten wir nur sehr einfache Spielzeuge » (Enfants, nous n'avions que des jouets très simples) ${ }^{39}$, «In den Westen durften nur Rentner » (Seuls les retraités pouvaient se rendre à l'ouest) ${ }^{40} \ldots$ Rarement le narrateur-auteur intervient pour préciser l'intention de son écriture. Une seule fois, dans le chapitre intitulé « Réalisme socialiste » de Mein erstes T-Shirt, dont le seul nom suffit à trahir un contenu plus politique que prosaïque, l'écrivain se positionne avec une certaine distance ironique en tant qu'instance sociale essentielle à la conservation $\mathrm{du}$ passé socialiste: "Einige dieser Erlebnisse habe ich zusammengetragen, um deutlich zu machen, welcher Schatz von Volksweisheit uns verlorengehen kann.» (J'ai rassemblé certains de ces faits vécus pour montrer quel trésor de sagesse populaire nous risquons de perdre ${ }^{41}$. Dans ce bref paragraphe, le lecteur est forcé de reconnaitre l'auteur derrière «le médecin qui recueille l'héritage oral» (Nur ab und $\mathrm{zu}$ findet sich auf den Stationen der Inneren Medizin ein Zeitgenosse, der im Sinne der mündlichen Überlieferung Erstaunliches berichten $k a_{n}{ }^{42}$ ). Rarement la volonté de soustraire à l'oubli le passé de la RDA est exprimée avec autant de clarté. En dépit du ton détaché de l'ensemble du texte, on est frappé par la double mission dont l'auteur se sent investi : expliquer et témoigner. Wladimir Kaminer utilise dans sa préface au premier ouvrage autobiographique de Jakob Hein une image insolite mais parfaitement en accord avec le caractère particulièrement systématique d'une écriture minutieusement ancrée dans le détail du quotidien : «Jakob Hein ist ein Staubsauger. Konsequent und unermüdlich saugt er alles auf, was er um sich herum sieht, und verarbeitet die eigene und fremde Realität in akkurate, zweieinhalb Seiten lange Geschichten. » (Jakob Hein est un aspirateur. Inlassablement et méthodiquement, il aspire tout ce qu'il voit autour de lui et transforme sa propre réalité et celle des autres en histoires soignées de deux pages et demie) ${ }^{43}$. Mais cette réalité est d'abord celle d'une Allemagne disparue dont l'auteur se réclame malgré lui. Dans Formen menschlichen Zusammenlebens, Jakob Hein se présente avec insistance comme l'Allemand de l'Est en visite à New York : "Während ich zum tausendsten Mal die üblichen Fragen beantwortete, wie der Fall der Berliner Mauer für mich gewesen sei und wie ich mich jetzt hier in Amerika fühle. » (Tandis que répondais pour la millième fois aux questions classiques : comment j'avais vécu la Chute du Mur de Berlin et comment je me sentais maintenant ici en Amérique) ${ }^{44}$. Dans Vielleicht ist es sogar schön, l'écrivain part même à la recherche de ses origines juives à travers l'histoire de sa famille maternelle. Une même quête des origines anime d'un souffle discret mais continu l'impérieux désir d'écriture qui trahit d'abord chez Jakob Hein un besoin vital de se positionner dans le présent. Il 
aura fallu au jeune écrivain le succès d'un triptyque familial au ton doux-amer pour pouvoir entrer de plain-pied dans l'univers de la fiction et renoncer à toute allusion référentielle: en 2006, Jakob Hein publie son premier «vrai» roman, dont le personnage principal se démarque ouvertement du modèle auctorial : Herr Jensen steigt aus est salué par la critique comme une authentique œuvre de fiction ${ }^{45}$.

\section{Conclusion}

La littérature du "moi» se décline en de nombreuses catégories: mémoires, confessions, souvenirs, essais, carnets... qui posent l'être intime comme objet d'analyse. Or en dépit de sa nature autobiographique, l'écriture chez Jakob Hein ne correspond pas à une plongée introspective dans un univers élégiaque, loin s'en faut. Le respect du pacte autobiographique cependant indique bien la nature autoréférentielle de l'écriture et fonde aussi la question identitaire de l'écriture. Écrivain des origines, l'autobiographe recherche l'origine de soi. "Toute autobiographie est une auto interprétation » (Jean Starobinski, Poétique ${ }^{\circ}{ }^{\circ}$, p. 258). Écriture du dévoilement de l'intériorité, l'écriture autobiographique répond à une fonction heuristique essentielle. Après avoir examiné le caractère autobiographique, familial et testimonial des débuts littéraires de Jakob Hein, force est de constater la fonction identitaire prédominante d'une écriture des origines qui mêle réalité personnelle et écriture fictionnelle. Depuis l'apparition du terme «autofiction » dans les années 1970, les débats font rage autour de cette nouvelle stratégie de narration, dont les frontières avec l'autobiographie sont encore mal délimitées et incertaines. S'inscrivant dans cette réflexion générique, on peut s'interroger à propos des premières œuvres de Jakob Hein sur l'oscillation de son écriture entre ces deux pôles. Mais avec Herr Jensen steigt aus, Hein semble avoir dépassé le stade de l'écriture autobiographique identitaire et livre un véritable roman, dont l'univers fictionnel s'est entièrement émancipé du poids de l'histoire familiale.

\section{NOTES}

1. Christoph Hein fait partie, aux côtés de Christa Wolf et Volker Braun, des écrivains majeurs de l'ancienne RDA dont la carrière se poursuit dans le contexte de l'Allemagne unifiée. Parmi ses textes les plus connus on peut citer : Der fremde Freund/Drachenblut (Berlin, Aufbau, 1982), Horns Ende (Berlin, Aufbau, 1985), Landnahme (Francfort, Suhrkamp, 2004).

2. Carola Hähnel-Mesnard: «La rupture dans la vie - Les récits de la jeune génération d'Allemands de l'Est entre témoignage et fiction ", in: Allemagne d'aujourd'hui, n 169 , juilletseptembre 2004, p. 146-159.

3. Il faudra attendre 2006 et la publication de Herr Jensen steigt aus (München, Piper) pour que l'œuvre de Jakob Hein bascule résolument dans la fiction avec un premier roman identifié en tant que tel dès la couverture.

4. «Im wirklichen Leben ist Jakob Hein Arzt und lebt mit seiner Familie in Berlin » (Mein erstes Tshirt, München, Piper, 2001). On trouve la même phrase à une variante près dans l'édition du 
deuxième ouvrage de l'auteur : «Im eigentlichen Leben ist Jakob Hein Arzt und lebt mit seiner Familie in Berlin » (Formen menschlichen Zusammenlebens, München, Piper, 2003).

5. Cf. Article de Henryk M. Broder, in : Der Spiegel, 44/2001, 29.10.2001, p. 192 : «Der Arzt und Schriftstellersohn Jakob Hein schildert in seinem Erzähldebüt «Mein erstes T-Shirt» muntere Episoden aus einer düsteren DDR-Jugend [...] 'wie es damals wirklich war' oder 'wie es wirklich gewesen sein könnte' ».

6. «Jakob Heins Episodentext Mein erstes T-Shirt ist allerdings keine autobiographische Erinnerungsliteratur. Er ironisiert vielmehr literarische Muster und stellt als Verarbeitung medialisierter Erinnerung einen selbstreferentiellen Endpunkt der literarischen und kollektiven DDR-Erinnerungen dar. » Elke Brüns, «Erinnerung im Zeichen von Pop in Jakob Heins 'Mein erstes T-Shirt' ", in: Akten des XI. Internationalen Germanistenkongresses Paris 2005, Jahrbuch für Internationale Germanistik, Band 86, Bern, Berlin, Bruxelles, Frankfurt am Main, New York, Oxford, Wien : Peter Lang, p. 409-414.

7. «Das Leben steckt voller Geheimnisse und unser jugendlicher Held Jakob Hein macht sich daran, sie zu lüften. » (Mein erstes T-Shirt, München, Piper, 2001, 5. Auflage 2005).

8. Sur la définition du rôle et de la fonction de la préface allographe, voir G. Genette, Seuils, Paris, Ed. Seuil, 1987, réédition Points, 2001, p. 245-247.

9. Wladimir Kaminer, « Mein erster Jakob Hein », in : Mein erstes T-Shirt, op. cit., p. 5-7 : "Jakob Hein ist ein geheimnisvoller Mensch. [...] Diese Vielseitigkeit von Jakob könnte die breiten Lesermassen verwirren und für alle möglichen Spekulationen über den Ich-Erzähler sorgen. Deshalb möchte ich schon im Vorfeld mit Hilfe dieses Vorworts klären, wer Jakob Hein wirklich ist. "

10. «Nach seinem hinreißenden und überaus erfolgreichen Debüt Mein erstes T-Shirt zieht es Jakob Hein in die Neue Welt» (Formen menschlichen Zusammenlebens, München, Piper, 2003, Ungekürzte Taschenbuchausgabe 2005).

11. «Jakob Hein, geboren 1971 in Leipzig, veröffentlichte 2001 sein autobiographisches Buch Mein erstes T-Shirt. » (Antrag auf ständige Ausreise und andere Mythen aus der DDR, München, Piper, 2007).

12. Philippe Lejeune : Le pacte autobiographique, Paris, Seuil, 1970.

13. Cf. Mein erstes T-Shirt, op. cit., p. 26 : « Dies schrieb Dir ein Dein Schlulfreund Jakob Hein.».

14. Cf. Elke Brüns, op. cit., p. 411 : "So sind die Erinnerungen bei Hein ironisch eingesetzte Nachwendetopoi, die als mediatisierte Erinnerung im Zeichen von Pop fungieren. »

15. Cf. Article de Henryk M. Broder, op. cit., p. 194 : «Viele seiner Miniaturen lesen sich wie Krankengeschichten ».

16. Ibid., p. 194 : «Er könnte, sagt Hein, «kein Buch über die DDR schreiben, nur darüber, was ich in der DDR erlebt habe». »

17. Cf. Préface éditoriale, Jakob Hein, Herr Jensen steigt aus, Piper, München, 2006, Taschenbuchausgabe 3. Auflage 2008.

18. Cf. Jörg Magenau, Deutschland-online, www.magazine-deutschland.de/issue/Lit_5-04.

19. Cf. Philippe Gasparini : Est-il je?, Paris, Seuil, 2004, p. 10. «Les genres narratifs en première personne, qui apparurent et se développèrent $d u \mathrm{XVII}^{\mathrm{e}}$ au XIX ${ }^{\mathrm{e}}$ siècle (Mémoires, lettres, journal intime, autobiographie) étaient rangés, non seulement par les poéticiens, mais aussi par les auteurs, dans le domaine prosaïque de la chronique, du particulier, de la non-littérature . » Dans l'introduction à son étude sur l'autofiction, Gasparini souligne également le travail accompli par Philippe Lejeune sur l'autobiographie, qui « a remis en question l'exclusion du récit référentiel du champ littéraire » (p. 11).

20. «Aucun art n'envisage un cas individuel », Aristote : Rhétorique, Paris, Livre de Poche, 1991, p. 86.

21. Mein erstes T-Shirt, op. cit., p. 9. 
22. Cf. Article de Henryk M. Broder, op. cit., p. 194 : « Hein ist ein Psychiater, der sich schreibend selbst therapiert: 'Wenn ich über etwas nicht nachdenken möchte, dann schreibe ich es auf.' ».

23. Vielleicht ist es sogar schön, op. cit., p. 18.

24. Cf. Formen menschlichen Zusammenlebens, op. cit., p. 24 : «Außerdem wohnten wir beide bei unseren ständig anwesenden Eltern ».

25. Mein erstes T-Shirt, op. cit., p. 25.

26. Ibid., p. 124-125.

27. Cf. Article de Henryk M. Broder, op.cit, p. 192.

28. Cf. Formen menschlichen Zusammenlebens, op.cit., p. 7-9.

29. Ibid., p. 19 : « Lesen war also zu beschwerlich, und deshalb hatte ich Zeit, statt dessen vor dem Einschlafen wichtige Entscheidungen zu treffen.» (Il était trop malaisé de lire, c'est pourquoi j'eus finalement le temps de mûrir des décisions importantes avant de m'endormir).

30. Ibid., p. 17-18.

31. Ibid., p. 25.

32. Ibid., p. 149.

33. Cf. Mein erstes T-Shirt, op. cit., p. 14, 17, 73, 96, 110.

34. Vielleicht ist es sogar schön, op. cit., Préface éditoriale : Zu diesem Buch.

35. Cf. Article de Henryk M. Broder, op. cit, p. 194 : « Nur kurz hatte Hein überlegt, ob er sich ein Pseudonym zulegen soll, um nicht ständig die Frage zu hören: Wie ist es für einen jungen Autor, der Sohn eines bekannten Schriftstellers zu sein? Doch dann fand er, «dass der Name Hein angenehm ist, weil er kurz ist und leicht zu merken. »

36. Cf. Carola Hähnel-Mesnard : «La rupture dans la vie», op. cit., p. 147 : le terme «Generation Mauerfall » désigne les auteurs encore adolescents au moment de la chute du Mur et conscients $\mathrm{du}$ fonctionnement du système, mais sans devoir prendre encore de décision déterminante pour leur avenir. Pour eux, c'est la rupture d'un changement de système politique et les expériences vécues dans le processus de transition, intervenant lors du passage décisif de l'enfance à l'âge adulte, qui apparaissent comme structurantes.

37. Cf. Elke Brüns, op. cit., p. 410.

38. Mein erstes T-Shirt, op. cit., p. 150.

39. Ibid., p. 124, 85, 70.

40. Formen menschlichen Zusammenlebens, op. cit., p. 7.

41. Mein erstes T-Shirt, op. cit., p. 92.

42. Ibid., p. 92.

43. Wladimir Kaminer, « Mein erster Jakob Hein », op. cit., p. 7.

44. Cf. Formen menschlichen Zusammenlebens, op. cit., p. 104.

45. Cf. Article de Jörg Magenau, in : taz.de, 18.02.2006 : «Jakob Hein, Jahrgang 1971, hat sich zu dem Tonfall, der dafür nötig ist, in drei eher autobiografisch angelegten Büchern vorgearbeitet. [...] erst «Herr Jensen steigt aus» ist ganz und gar literarisch. » (Jakob Hein, né en 1971, a trouvé le ton juste en travaillant d'abord à trois romans d'inspiration autobiographique, mais ce n'est qu'avec Herr Jensen steigt aus qu'il fait œuvre littéraire d'un bout à l'autre). 


\section{RÉSUMÉS}

Depuis ses débuts littéraires sur le ton léger de l'autodérision avec Mein erstes T-Shirt (Mon premier T-shirt) (Piper, 2001) et Formen menschlichen Zusammenlebens (Formes de cohabitation humaine) (Piper, 2003) jusqu'à un premier roman sobre et émouvant consacré à la mort de sa mère Vielleicht ist es sogar schön (Peut-être même que c'est beau) (Piper, 2004), Jakob Hein ne cesse de ressasser, commenter et interroger l'histoire familiale à travers sa fiction. Né en 1971 à Leipzig et fils d'un écrivain renommé, il fait partie des jeunes auteurs de l'Est dont l'écriture se nourrit pour une part essentielle du terreau familial. Le dialogue entre les générations, chez ce jeune écrivain de l'Allemagne réunifiée, est immanent et constitutif d'une écriture dont le caractère à la fois autobiographique, familial et testimonial ne laisse pas indifférent: une écriture des origines qui mêle réalité personnelle et écriture fictionnelle et dont la fonction identitaire dans le cadre des débuts littéraires de l'auteur est patente. Mais avec Herr Jensen steigt aus (Piper, 2006), Hein semble avoir dépassé le stade de l'écriture autobiographique identitaire et livre un véritable roman dont l'univers fictionnel s'est entièrement émancipé du poids de l'histoire familiale.

In seinem Erzähldebüt Mein erstes T-Shirt (Piper, 2001) wie später in Formen menschlichen Zusammenlebens (Piper, 2003) bis hin zum stillen und einfühlsamen Erinnerungsbuch über den frühen Tod der Mutter Vielleicht ist es sogar schön (Piper, 2004) schildert der praktizierende Berliner Arzt und Schriftstellersohn Jakob Hein systematisch und konsequent Episoden aus dem eigenen Leben, sei es in der düsteren DDR-Vergangenheit oder in der gegenwärtigen kapitalistischen Wunderwelt. Der Dialog der Generationen, bzw. die Bedeutung der Familie in Heins Debütwerk scheint dabei als entscheidendes Element einer identitätsstiftenden Literatur $\mathrm{zu}$ fungieren. Auf der Suche nach Orientierungslinien beleuchtet Jakob Hein zunächst hauptsächlich Biografisches. Für seinen ersten Roman Herr Jensen steigt aus (Piper, 2006) hat jedoch der junge Autor erstmals eine fiktive literarische Figur geschaffen und somit die schwerwiegende Familiengeschichte anscheinend überwunden.

\section{INDEX}

oeuvrecitee Formen menschlichen Zusammenlebens, Mein erstes T-shirt, Vielleicht ist es sogar schön

\section{AUTEURS}

\section{HÉLÈNE YÈCHE}

Université de Poitiers 